\title{
Tenckhoff tunneled peritoneal catheter placement in the palliative treatment of malignant ascites: technical results and overall clinical outcome
}

\author{
Geert Maleux ${ }^{1}$, Inge Indesteege ${ }^{1}$, Annouschka Laenen², Chris Verslype ${ }^{3}$, Ignace Vergote ${ }^{4}$, \\ Hans Prenen ${ }^{3}$ \\ ${ }^{1}$ Department of Radiology, University Hospitals Leuven, Belgium \\ ${ }^{2}$ Department of Biostatistics and and Statistical Bioinformatics, KU Leuven and Universiteit Hasselt, Belgium \\ ${ }^{3}$ Department of Digestive Oncology, University Hospitals Leuven, Belgium \\ ${ }^{4}$ Department of Gynaecology, University Hospitals Leuven, Belgium
}

Radiol Oncol 2016; 50(2): 197-203.

Received 13 October 2015

Accepted 20 December 2015

Correspondence to: Geert Maleux, M.D., Ph.D., Department of Radiology, University Hospitals Leuven, Herestraat 49, B-3000 Leuven; Belgium. Phone: +32 163437 82; Fax: +32 163437 65; E-mail: geert.maleux@uzleuven.be

Disclosure: The authors have no conflicts of interest to disclose.

Background. To assess the technical and clinical outcome of percutaneous insertion of tunneled peritoneal catheters in the palliative treatment of refractory malignant ascites and to determine the safety and feasibility of intraperitoneal administration of cytotoxic drugs through the tunneled catheter.

Materials and methods. Consecutive patients palliatively treated with a tunneled peritoneal catheter to drain the malignant ascites were identified. Patients' medical history, procedural and clinical follow-up data, including complications and estimated survival, were reviewed. Additionally, a sub analysis of the patients with widespread ovarian cancer and refractory ascites treated with or without intraperitoneal administration of cytotoxic drugs was made.

Results. In all 94 patients it was technically feasible to insert the peritoneal drainage catheter and to drain a median of $3260 \mathrm{cc}$ (range $100 \mathrm{cc}-8500 \mathrm{cc}$ ) of malignant ascitic fluid. Post procedural complications included catheter infection ( $n=2 ; 2 \%)$, fluid leakage around the entry site $(n=4 ; 4 \%)$, catheter occlusion $(n=2 ; 2 \%)$, sleeve formation around the catheter tip $(n=1 ; 1 \%)$ and accidental loss of the catheter $(n=1 ; 1 \%)$. There was no increase in catheter infection rate in patients treated with or without intraperitoneal administration of cytotoxic drugs. Median overall survival after catheter insertion is 1.7 months.

Conclusions. Percutaneous insertion of a tunneled Tenckhoff catheter for the palliative drainage of malignant ascites and intraperitoneal infusion of cytotoxic drugs is feasible and associated with a very low complication rate, including catheter infection. These tunneled peritoneal lines are beneficial for symptomatic palliative treatment of refractory ascites and allow safe intraperitoneal chemotherapy.

Key words: peritoneal catheter; malignant ascites; palliation

\section{Introduction}

Malignant ascites is a manifestation of terminal metastatic disease with a life expectancy ranging from 1 to 4 months; the ascitic fluid production is usually associated with peritoneal tumours, lymphangitic carcinomatosis, lymphatic obstruction, encasement of the portal vein by a tumour causing prehepatic portal hypertension, or a combination of these pathophysiological mechanisms. ${ }^{1-3}$ Clinically, these patients suffer from abdominal distension, early satiety, shortness of breath, fatigue or gastrointestinal symptoms such as nausea and vomiting. Medical treatment, including diuret- 
ics, have little or no effect on malignant fluid accumulation and the standard treatment for these patients was repeated paracentesis, despite the risks of infection, haemorrhage or bowel wall injury and the need for frequent trips to the hospital. ${ }^{4}$ In the past decade, alternative drainage options intended to avoid repetitive punctures, have been tested and used. These drainage techniques include internal drainage like peritoneo-venous ${ }^{5}$, peritoneo-gastric $^{6}$ and peritoneo-cystic ${ }^{7}$ shunting and external drainage techniques requiring the placement of an indwelling, tunneled peritoneal drainage or portcatheter. ${ }^{2,3,5,8-13} \mathrm{An}$ important disadvantage of external drainage is albumin loss, which may need to be considered in deciding between external drainage and internal shunts. Most of the experience with indwelling drainage catheters has been described with the PleurX catheter; this monocuffed catheter was initially designed for drainage of malignant pleural effusions but it can also be used for drainage of malignant peritoneal fluid., ${ }^{3,-11}$ Additionally, if this type of indwelling tunneled catheter is used, it is recommended to use vacuum bottles for adequate drainage.

In this study we retrospectively analysed the technical feasibility and safety of the insertion of a Tenckhoff peritoneal tunnelled catheter. Additionally, the overall clinical outcomes in this patient population were analysed and finally we evaluated the feasibility and safety of intraperitoneal chemotherapy delivery through the Tenckhoff catheter in patients with widespread ovarian cancer and refractory ascites using catumaxomab.

\section{Materials and Methods}

\section{Patients and study design}

A retrospective analysis was carried out on consecutive patients in whom a Tenckhoff tunnelled peritoneal catheter was inserted percutaneously for the management of refractory malignant ascites in the authors' institution between March 2006 and January 2013. The inclusion criteria for catheter placement were symptomatic, malignant ascites refractory to conservative and medical management in patients with widespread metastatic disease; haemostatic parameters allowing small skin incisions and subcutaneous tunnelling; absence of compartmentalization of the malignant ascitic fluid. Active infection is considered as an exclusion criterion for catheter insertion. Refractory malignant ascites is defined as ascites in patients with widespread metastatic disease in whom the ascites cannot be mobilized by conservative or medical therapies. Patients' history, procedural and post-procedural data were documented based on the patients' hospital electronic medical records and after telephone calls with the patients' general practitioners.

The patients gave informed consent before the start of the interventional procedure and institutional review board approval was obtained for this retrospective study analysis.

\section{Interventional procedure of Tenckhoff catheter placement}

Patients were referred to the interventional radiology department after discussion between the attending interventional radiologist and medical or surgical oncologist. Patient preparation included a bedside ultrasound for evaluation of the amount of ascites and more specifically for evaluation of a window of ascitic fluid at the intraperitoneal puncture site. The preferred intraperitoneal puncture site was near the midline, inferior and to the right of the umbilicus; if no ascitic fluid window was identified in that area, a left-sided infra-umbilical puncture site was prepared with a tunnel area to the left flank. Laboratory analysis included acceptable haematological parameters for tunneled catheter insertion, including a platelet count of at least 50,000/L, a haemoglobin level $>8 \mathrm{~g} / \mathrm{dL}$ and an International Normalized Ratio (INR) of less than 1.5. Tenckhoff tunneled peritoneal drainage catheter insertion was performed under sterile conditions in the interventional radiology suite.

After standard surgical preparation, local anaesthesia of the puncture site and the subcutaneous tunnel area was administered with $30 \mathrm{~mL}$ of lidocaine hydrochloride (Linisol 2\%, B. Braun, Diegem, Belgium). No other sedation or prophylactic antibiotic medication was administered; a $2 \mathrm{~cm}$ skin incision was made near the midline, inferior and to the right (or left) of the umbilicus and ultrasoundguided puncture of the malignant ascitic fluid was carried out using an 18 gauge $(\mathrm{G})$ sheathed needle (Surflo I.V. Catheter, Terumo Europe, Leuven, Belgium) (Figure 1A). A 0.035 inch hydrophilic guide wire (HydroSteer, St-Jude Medical, St-Paul, MN, USA) was introduced into the peritoneal cavity using a 0.035 inch 4 French (F) Cobra catheter (Slip-cath, Cook Medical, Bjaeverskov, Denmark) positioned in the pelvis (Figure 1B). This was then exchanged for a 0.035 inch stiff guide wire (Amplatz, Cook Medical, Bjaeverskov, Denmark) (Figure 1C). Over the stiff guide wire the punc- 


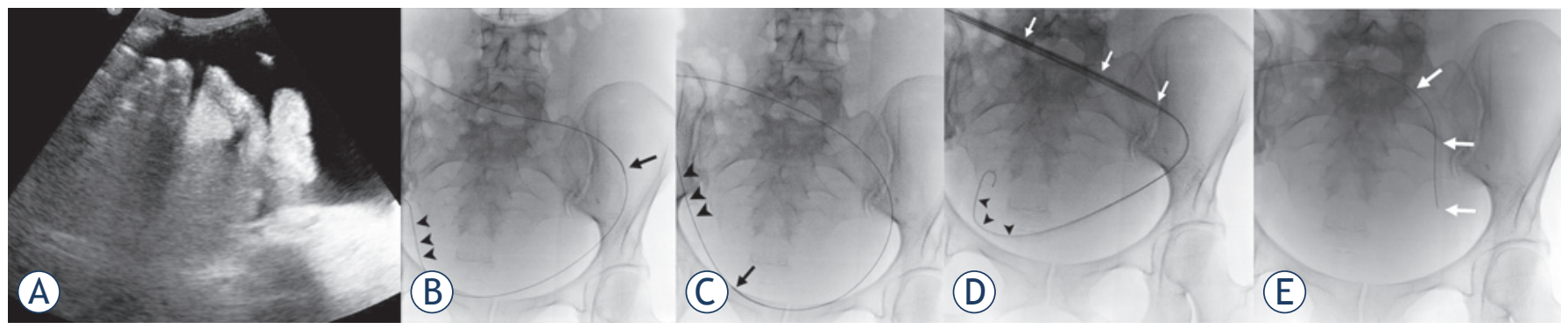

FIGURE 1. (A) Ultrasound-guided puncture of the ascitic fluid. The tip of the puncture needle (white arrow) is located within the fluid, far from intestinal or other abdominal structures. (B) Using a $4 \mathrm{~F} \mathrm{Cobra} \mathrm{catheter} \mathrm{(black} \mathrm{arrow),} \mathrm{the} \mathrm{hydrophilic} \mathrm{guide} \mathrm{wire} \mathrm{(arrowheads)} \mathrm{is} \mathrm{navigated} \mathrm{to} \mathrm{the} \mathrm{lower} \mathrm{portion} \mathrm{of}$ the pelvis. (C) The hydrophilic guide wire is exchanged for a stiff Amplatz wire (arrowheads) on the Cobra catheter (arrow). (D) The 15F peel-away sheath (white arrows) is introduced into the peritoneal cavity over the stiff Amplatz wire (arrowheads). (E) The Tenckhoff catheter (white arrows) is introduced through the $15 \mathrm{~F}$ peel-away sheath into the peritoneal cavity.

ture tract was dilated using a $8 \mathrm{~F}$ dilator (Cook Medical, Bjaeverskov, Denmark) and finally a 15F peel-away introducer (PTFE-peel-Apart, BARD Benelux, Olen, Belgium) was inserted (Figure 1D). The Tenckhoff peritoneal drainage catheter (Argyle peritoneal dialysis catheter, Covidien, Mansfield, MA, USA) with the Cobra catheter inside was introduced over the stiff guide wire into the peritoneal cavity and positioned in a curved position in the lower pelvic region (Figure 1E). The Tenckhoff catheter is made of translucent silicone rubber tubing containing a radio-opaque stripe. The total length of the $15 \mathrm{~F}$ catheter is $47 \mathrm{~cm}$ and the inner diameter is $2.6 \mathrm{~mm}$. The intraperitoneal part of the catheter contains small fenestrations over a length of $15 \mathrm{~cm}$ (Figure 2). The cuffed end of the Tenckhoff catheter is tunnelled to the right (or left) flank using a metallic tunnelling device (Argyle Faller Tunneling device, Covidien, Mansfield, MA, USA) and exteriorized $7 \mathrm{~cm}$ lateral to the peritoneal entry site. Finally, the small cutaneous incisions are sutured and the external part of the tunnelled catheter is connected to a drainage bag (3L Empty Bag System II, Baxter Healthcare, Zurich, Switzerland) using a sterile connecting device (Connection Shield System II with Povidone-Iodine Solution, Baxter Healthcare, Zurich, Switzerland) to begin drainage.

The intraperitoneal chemotherapy infusion technique was performed using a catumaxomabbased regimen as described by Baumann et al. ${ }^{14}$ Briefly, catumaxomab (Removab ${ }^{\circledR}$, Neovii Biotech, Waltham, MA, USA) $10 \mu \mathrm{g}, 20 \mu \mathrm{g}, 50 \mu \mathrm{g}$ and $150 \mu \mathrm{g}$ in $250 \mathrm{~mL}$ of $0.9 \% \mathrm{NaCl}$ physiologic solution was injected intraperitoneally through the Tenckhoff catheter, respectively at day $1,4,8$ and 11 of the treatment.

Patients were followed up until the end of the study (March 2013) or the patient's death.

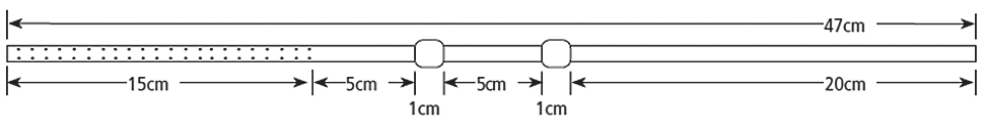

FIGURE 2. Schematic drawing of the Tenckhoff catheter: the intraperitoneal portion contains small fenestrations over a length of $15 \mathrm{~cm}$. Two cuffs with a length of $1 \mathrm{~cm}$ are positioned in the subcutaneous tissues.

\section{Statistical analysis}

Overall survival probabilities are estimated by the Kaplan-Meier method. The Wilcoxon test is used for testing survival differences between ovarian cancer patients with or without intraperitoneal chemotherapy treatment (IPCT). The prognostic value of primary pathology for survival is analysed using Cox proportional hazards models. Fisher's exact test is used for the association between intraperitoneal chemotherapy treatment and catheter infection.

All tests are two-sided. A 5\% significance level is assumed for all tests. All analyses have been carried out using SAS software, version 9.3 of the SAS System for Windows (SAS Institute Inc., Cary, NC, USA).

\section{Results}

\section{Patient demographics}

In 94 patients ( 27 men; $28.7 \%$ and 67 women; $71.3 \%$ ) with a mean age of 60.1 years (median 59.4 years; standard deviation 12.4 years) a tunnelled peritoneal Tenckhoff catheter was inserted for drainage of malignant ascites. Malignant ascites was associated with different types of metastatic cancer disease as summarized in Table 1. The category 'rest' included lung carcinoma $(n=2)$, multiple myeloma $(n=2)$ and myxoid liposarcoma $(n=1)$. 
TABLE 1. Type of primary cancer

\begin{tabular}{lcc}
\hline Primary malignant disease & Statistic & All \\
\hline Gynaecological cancer & $\mathrm{n} / \mathrm{N}(\%)$ & $41 / 94(43.6 \%)$ \\
Ovarian cancer & $\mathrm{n} / \mathrm{N}$ & $38 / 94$ \\
Endometrial cancer & $\mathrm{n} / \mathrm{N}$ & $3 / 94$ \\
Hepatobiliary cancer & $\mathrm{n} / \mathrm{N}(\%)$ & $24 / 94(25.5 \%)$ \\
Pancreatic cancer & $\mathrm{n} / \mathrm{N}$ & $11 / 94$ \\
Cholangiocarcinoma & $\mathrm{n} / \mathrm{N}$ & $12 / 94$ \\
Hepatocellular carcinoma & $\mathrm{n} / \mathrm{N}$ & $1 / 94$ \\
Gastrointestinal cancer & $\mathrm{n} / \mathrm{N}(\%)$ & $11 / 94(11.7 \%)$ \\
Colorectal cancer & $\mathrm{n} / \mathrm{N}$ & $6 / 94$ \\
Gastric cancer & $\mathrm{n} / \mathrm{N}$ & $3 / 94$ \\
Small bowel neuroendocrine cancer & $\mathrm{n} / \mathrm{N}$ & $2 / 94$ \\
Breast cancer & $\mathrm{n} / \mathrm{N}(\%)$ & $13 / 94(13.8 \%)$ \\
Rest & $\mathrm{n} / \mathrm{N}(\%)$ & $5 / 94(5.3 \%)$ \\
\hline
\end{tabular}

TABLE 2. Paracenteses prior to Tenckhoff catheter placement

\begin{tabular}{lcc}
\hline Number of paracenteses & Statistic & All \\
\hline 0 & $\mathrm{n} / \mathrm{N}(\%)$ & $19 / 94(20.2 \%)$ \\
1 & $\mathrm{n} / \mathrm{N}(\%)$ & $20 / 94(21.3 \%)$ \\
2 & $\mathrm{n} / \mathrm{N}(\%)$ & $16 / 94(17.0 \%)$ \\
3 & $\mathrm{n} / \mathrm{N}(\%)$ & $15 / 94(16.0 \%)$ \\
4 or $>4$ & $\mathrm{n} / \mathrm{N}(\%)$ & $24 / 94(25.5 \%)$ \\
\hline
\end{tabular}

TABLE 3. Kaplan-Meier estimates for overall survival since Tenckhoff insertion at specific follow-up times (+95\% confidence interval)

\begin{tabular}{lccc}
\hline Months since Tenckhoff insertion & \% Survival & Lower limit & Upper limit \\
\hline 3 & 30.0 & 20.9 & 39.6 \\
6 & 18.0 & 10.8 & 26.8 \\
12 & 7.7 & 3.2 & 14.5 \\
18 & 2.6 & 0.3 & 9.9 \\
24 & 2.6 & 0.3 & 9.9 \\
\hline
\end{tabular}

The number of paracenteses prior to Tenckhoff catheter insertion is indicated in Table 2; overall, patients underwent a mean of 3.4 paracenteses (median: 2.0; standard deviation: 5.6; range: $0-44$ paracenteses). In 15 out of 94 patients (16\%) intraperitoneal chemotherapy treatment (IPCT) with catumaxomab was given; these 15 patients suffered from widespread metastatic ovarian cancer associated with refractory malignant ascites.

\section{Technical outcome}

In all patients (100\%), the Tenckhoff tunnelled peritoneal drainage catheter was successfully inserted; in 90 patients $(96 \%)$, the Tenckhoff catheter was tunnelled subcutaneously into the right flank, in the remaining 4 patients ( $4 \%$ ) the peritoneal access was made in the left para and infraumbilical region and the catheter was tunnelled into the left flank. Once the Tenckhoff catheter was in place, a median of 3,260 cc (range $100 \mathrm{cc}-8,500 \mathrm{cc}$ ) of malignant ascitic fluid was drained.

\section{Clinical outcome}

Clinical follow-up was available for 90 patients; 4 patients $(4.2 \%)$ were lost to follow-up.

Two patients $(2.1 \%)$ presented with a clinical suspicion of catheter infection, including fever, painful cutaneous and subcutaneous tunnel infection, but without clear signs of peritonitis, 36 \& 40 days respectively after initial catheter placement. One of these two patients was also treated with intraperitoneal chemotherapy infusions. Other minor complications included ascitic fluid leakage around the entry point of the catheter in the right flank $(n=4 ; 4 \%)$, catheter occlusion $(n=2 ; 2 \%)$ and sleeve formation around the tip of the catheter resulting in insufficient drainage $(n=1 ; 1 \%)$. Management of these complications included extra skin sutures around the catheter entry point $(n=4)$, catheter removal $(n=1)$ or catheter flushing $(n=2)$ respectively.

In another three patients (3\%), initially presenting with malignant ascites related to breast carcinoma $(n=1)$, endometrial carcinoma $(n=1)$ and ovarian carcinoma $(n=1)$, the Tenckhoff catheter was removed after 111, 134 and 39 days respectively, owing to regression of ascitic fluid production. Another patient accidentally lost the catheter 11 days after initial placement.

Five out of 90 patients $(5.3 \%)$ were still alive at the end of the study (March 2013); the remaining 85 patients (90.4\%) died before March 2013. The time interval until end of follow-up or the patient's death was a mean of 3.41 months (median 1.7 months; standard deviation: 4.73; min: 0.03, $\max$ 25.7 months).

Kaplan-Meier estimates for overall survival after Tenckhoff insertion is summarized in Figure 3 and Table 3, showing an estimated survival at 3 and 6 months of nearly $30 \%$ and $18 \%$ respectively. Further, a more detailed analysis of survival after Tenckhoff catheter insertion is made based on the underlying 


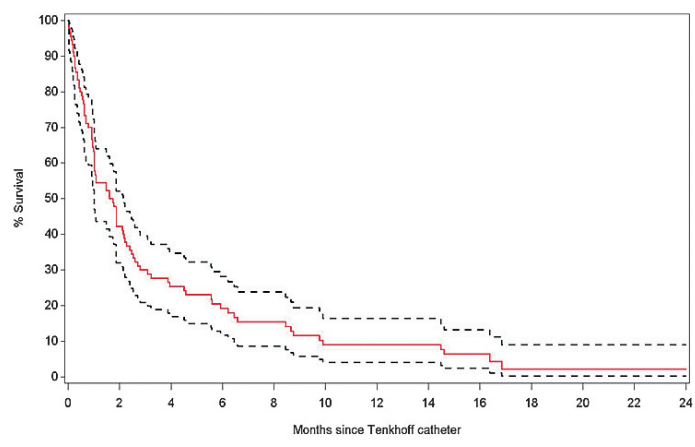

\begin{tabular}{|rrrrrrrrrrrrr|}
\hline Number at risk & & & & & & & & & & \\
90 & 38 & 22 & 15 & 12 & 7 & 7 & 7 & 4 & 1 & 1 & 1 & 1 \\
\hline
\end{tabular}

FIGURE 3. Overall survival after Tenckhoff catheter insertion with 95\% confidence limits.

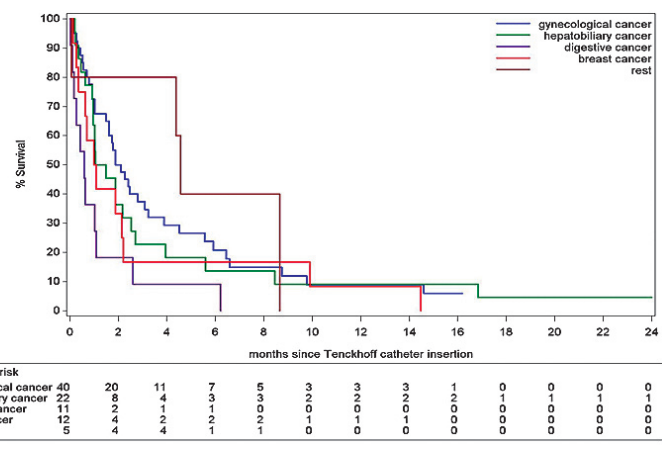

FIGURE 4. Overall estimated survival for different cancer types after Tenckhoff catheter insertion.

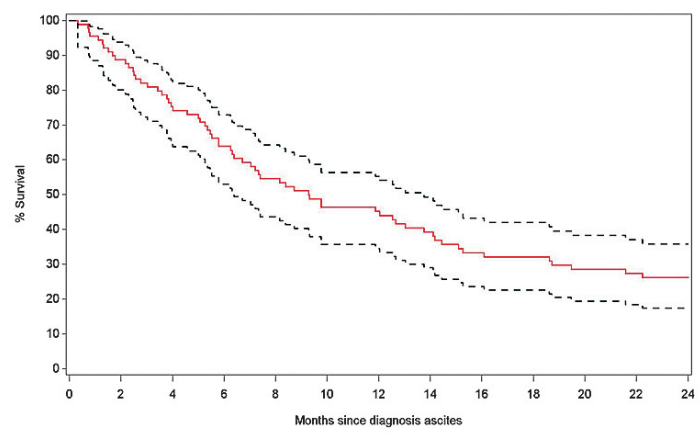

\begin{tabular}{|rrrrrrrrrrrrr|}
\hline Number at risk & & & & & & & & & & & & \\
89 & 79 & 67 & 55 & 47 & 39 & 38 & 33 & 28 & 27 & 24 & 23 & 22 \\
\hline
\end{tabular}

FIGURE 5. Overall estimated survival since clinical diagnosis of malignant ascites with $95 \%$ confidence limits.

type of cancer. We analysed five categories of underlying aetiologies: gynaecological cancers $(n=40)$ including ovarian and endometrial cancers; hepatobiliary cancers $(n=22)$ including pancreatic cancer, cholangiocarcinoma and hepatocellular carcinoma;
TABLE 4. Kaplan-Meier estimates for overall survival since clinical diagnosis of ascites at specific follow-up times (+ $95 \%$ confidence interval)

\begin{tabular}{lccc}
\hline $\begin{array}{l}\text { Months since clinical diagnosis } \\
\text { of ascites }\end{array}$ & \% Survival & Lower limit & Upper limit \\
\hline 3 & $82.2 \%$ & $72.6 \%$ & $88.7 \%$ \\
6 & $63.2 \%$ & $53.3 \%$ & $72.2 \%$ \\
12 & $44.7 \%$ & $34.1 \%$ & $54.7 \%$ \\
18 & $30.6 \%$ & $21.2 \%$ & $40.4 \%$ \\
24 & $24.7 \%$ & $16.2 \%$ & $34.1 \%$ \\
\hline
\end{tabular}

TABLE 5. Analysis of overall survival since clinical diagnosis of malignant ascites for different groups of cancers

\begin{tabular}{lcccc}
\hline & $\begin{array}{c}\text { Hazard } \\
\text { ratio }\end{array}$ & $\begin{array}{c}\text { Lower } \\
\text { limit }\end{array}$ & $\begin{array}{c}\text { Upper } \\
\text { limit }\end{array}$ & P-value \\
\hline Gynaecological cancers (reference) & - & - & - & 0.06 \\
Hepatobiliary cancers & 1.17 & 0.68 & 2.02 & 0.575 \\
Gastrointestinal cancers & 2.58 & 1.30 & 5.13 & 0.007 \\
Breast cancer & 1.42 & 0.73 & 2.74 & 0.299 \\
Rest & 0.71 & 0.25 & 1.99 & 0.511 \\
\hline
\end{tabular}

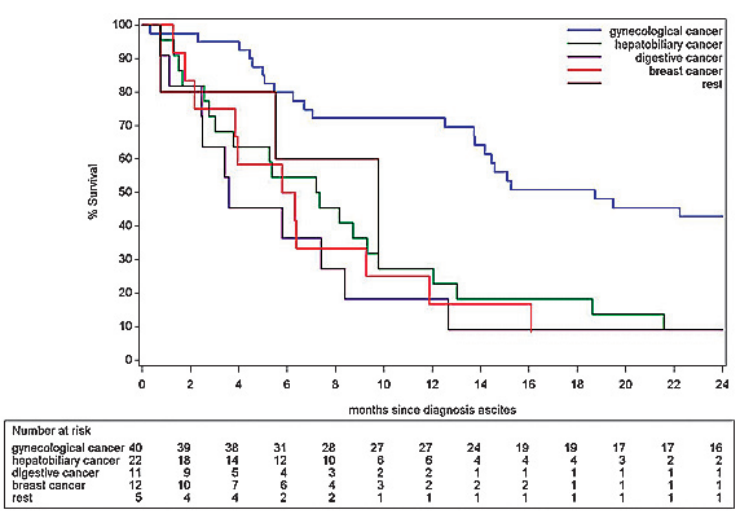

FIGURE 6. Overall estimated survival for different types of cancer since clinical diagnosis of malignant ascites.

gastrointestinal cancers $(\mathrm{n}=11)$ including colorectal cancer, gastric cancer and neuroendocrine tumours; breast carcinoma $(n=11)$ and rest $(n=5)$ including lung carcinoma $(\mathrm{n}=2)$, multiple myeloma $(\mathrm{n}=$ 2 ) and myxoid liposarcoma $(n=1)$; the survival of these different groups is summarized in Figure 4. Analysis suggests a difference in risk for early death after Tenckhoff catheter insertion according to the underlying cancer: patients with widespread gastrointestinal cancers and refractory malignant ascites have a higher risk for early death compared to the reference group of patients with widespread metastatic gynaecological cancers. 
TABLE 6. Survival analysis in patients with metastatic ovarian cancer and malignant ascites treated with or without intraperitoneal infusion of catumaxomab after Tenckhoff catheter insertion

\begin{tabular}{lccc}
\hline \multirow{2}{*}{ IPCT } & $\begin{array}{c}\text { Median survival } \\
\text { in months }\end{array}$ & \multicolumn{2}{c}{$95 \%$ confidence interval } \\
\cline { 3 - 4 } & 3.22 & Lower limit & Upper limit \\
\hline With IPCT & 1.61 & 1.61 & 6.58 \\
Without IPCT & & 0.69 & 2.40 \\
\hline
\end{tabular}

IPCT = intraperitoneal chemotherapy treatment

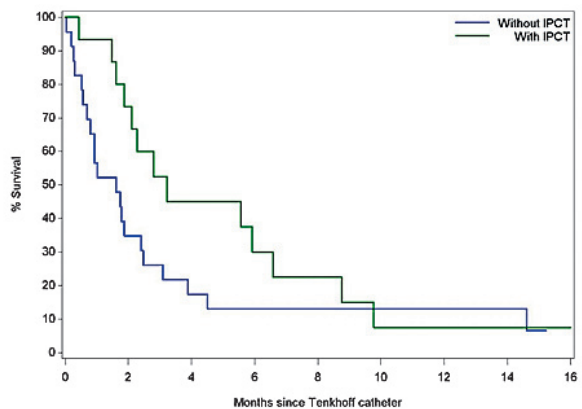

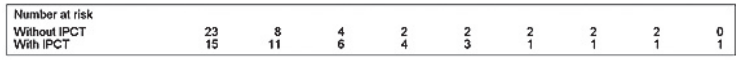

FIGURE 7. Overall estimated survival in patients with metastatic ovarian cancer associated with malignant ascites is better if intraperitoneal infusion of catumaxomab through the Tenckhoff catheter is carried out ( $p=0.02)$.

Overall survival after clinical diagnosis of malignant ascites demonstrates a 3 and 6 month estimated survival of $82.0 \%$ and $63.9 \%$ respectively, as shown in Table 4 and Figure 5. An analysis of the potential outcome differences between the five categories of cancer mentioned above was carried out and summarized in Table 5 and Figure 6. The risk analysis for early death after clinical diagnosis of malignant ascites also demonstrates a significant difference in survival for patients with malignant gynaecological cancer compared to patients with gastrointestinal cancers $(\mathrm{p}=0.007)$.

Tunnelled Tenckhoff catheters were inserted in a total of 38 patients presenting with metastatic ovarian cancer and malignant ascites. In 23 of these patients the Tenckhoff catheter was inserted solely for repeated drainage purposes. In the remaining 15 patients the Tenckhoff catheter was inserted for the purpose of drainage of malignant ascites and for the purpose of intraperitoneal infusion of a catumaxomab-based solution. Overall survival of the two sub-groups of patients (Table 6 and Figure 7) revealed better survival in the group with intraperitoneal infusion of catumaxomab $(\mathrm{p}=0.02)$.

\section{Discussion}

This study demonstrates a very high technical success rate $(100 \%)$ for tunnelled, peritoneal Tenckhoff catheter insertion in patients suffering from refractory malignant ascites, which is in line with experiences in other centres using the same ${ }^{2,15}$ or other types of tunnelled peritoneal catheters such as the PleurX-catheter ${ }^{3,8-11}$ or Medcomp catheter. ${ }^{16}$ Furthermore, subcutaneous insertion of port catheters has a $100 \%$ success rate ${ }^{12,13}$, although there are only a few reports covering a small number of included patients. The major difference between the PleurX catheter and the Tenckhoff or Medcomp catheter is the number of cuffs: the PleurX catheter has one cuff whereas the other two have two cuffs; the number of infection events with these different types of tunnelled catheters does not, however, seem to be different: we encountered two patients $(2 \%)$ with clinical signs of infection which is almost identical to the series with the PleurX catheter., ${ }^{9,11}$

The technique of tunnelled catheter insertion is essentially the same for the different types of peritoneal tunnelled catheters: percutaneous access to the peritoneal cavity is gained under ultrasound guidance using Seldinger technique and insertion of the catheter through a peel-away sheath can be performed blindly or under fluoroscopic guidance. When using these techniques, however, the position of the tip of the tunnelled catheter is not always predictable. Instead, we used a catheterbased technique (Cobra catheter and hydrophilic guide wire) to position the tip and the fenestrated area of the Tenckhoff catheter in the dependent portion of the peritoneal cavity (lower pelvic region) which might result in better drainage of the ascitic fluid later on, especially when the patient is in a sitting or supine position, although catheter tip migration after insertion is still possible especially in case of recurrent ascitic fluid accumulation associated with bowel and body movements in general.

Other post-procedural complications apart from infection are almost always minor complications and may include fluid leakage around the catheter entry point, catheter occlusion or accidental loss despite the presence of two cuffs. This very low rate of serious complications may suggest earlier referral for Tenckhoff catheter placement for the palliative drainage of malignant ascites resulting in patients' improved quality of life.

Importantly, this study also suggests the usefulness of the Tenckhoff catheter for intraperitoneal administration of chemotherapeutic agents such as catumaxomab without a significant increase in 
adverse, infectious events, although the number of patients treated with intraperitoneal chemotherapy infusion was small $(\mathrm{n}=15)$.

The life expectancy of patients with refractory malignant ascites is very poor, with a range from 1 to 4 months, which is in line with the overall results of this study, showing a median overall survival of 1.7 months. This short life expectancy mainly depends on the natural history of the underlying widespread malignancy and subsequently patients with a longer life expectancy associated with refractory malignant ascites, such as patients with gynaecological tumours, may also benefit from the tunnelled Tenckhoff catheter for a longer period compared to patients with more aggressive tumours such as gastrointestinal malignancies.

Finally, a sub-analysis of patients with refractory ascites and widespread malignant ovarian tumours reveals improved survival if catumaxomab is administered intraperitoneally $(p=0.02)$. This conclusion should be interpreted with caution, however, because this is a retrospective, singlecentre, non-randomized analysis including a small number of patients. Additionally, a multi-centre, randomized open-label phase IIa study was only able to demonstrate a slightly better therapeutic index in a high-dose catumaxomab regimen as compared to a low-dose regimen ${ }^{14}$ and other researchers found a non-significant survival benefit (110 days versus 81 days) if intraperitoneal administration of catumaxomab took place in patients with recurrent ovarian cancer. ${ }^{17}$

In conclusion, this study demonstrates that percutaneous image-guided insertion of a tunnelled Tenckhoff catheter in the peritoneal cavity is safe and effective for drainage of refractory malignant ascites, with a very low complication rate including catheter infection. The catheter is also an efficient and safe tool for intraperitoneal administration of cytotoxic drugs with no increase in peritonitis or other infectious adverse events. Finally, owing to the natural course of the underlying malignant tumor, patients with widespread metastatic gynaecological cancers and refractory ascites may benefit for a longer period from this interventional procedure than patients with other cancers and associated malignant ascites.

\section{References}

1. Mullard A, Bishop J, Jibani M. Intractable malignant ascites: An alternative management option. J Palliative Med 2011; 2: 251-3.

2. O'Neill M, Weissleder R, Gervais D, Hahn P, Mueller P. Tunneled peritoneal catheter placement under sonographic and fluoroscopic guidance in the palliative treatment of malignant ascites. Am J Roentgenol 2001; 177: 615-8.
3. Richard H, Coldwell D, Boyd-Kranis R, Murthy R, Van Echo D. Pleurx tunneled catheter in the management of malignant ascites. J Vasc Intervent Radiol 2001: 12: 373-5.

4. Belfort M, Stevens P, DeHaek K, Soeters R, Krige J. A new approach to the management of malignant ascites; a permanently implanted abdominal drain. Eur J Surg Oncol 1990; 16: 47-53.

5. Arai Y, Inaba Y, Sone M, Saitoh H, Takeuchi Y, Shioyama Y, et al. Plase I/ II study of transjugular transhepatic peritoneovenous venous shunt, a new procedure to manage refractory ascites in cancer patients: Japan Interventional Radiology in Oncology Study Group 0201. Am J Roentgenol 2011; 196: W621-W626.

6. Lorentzen T, Sengel $\varnothing v \mathrm{~L}$, Nolsøe CP, Khattar SC, Karstrup S, von der Maase H. Ultrasonically guided insertion of a peritoneo-gastric shunt in patients with malignant ascites. Acta Radiol 1995; 36:481-4.

7. Stehman F, Ehrlich C. Peritoneo-cystic shunt for malignant ascites. Gynecol Oncol 1984; 18: 402-7.

8. Rosenberg S, Courtney A, Nemcek A, Omary R. Comparison of percutaneous management techniques for recurrent malignant ascites. I Vasc Intervent Radiol 2004; 15: 1129-31.

9. Tapping C, Ling L, Razack A. PleurX drain use in the management of malignant ascites: safety, complications, long-term patency and factors predictive of success. Br J Radiol 2012; 85: 623-8.

10. Lungren M, Kim C, Stewart J, Smith T, Miller M. Tunneled peritoneal drainage catheter placement for refractory ascites: Single-center experience in 188 patients. J Vasc Intervent Radiol 2014; 24: 1303-8.

11. Courtney A, Nemcek A, Rosenberg S, Tutton S, Darcy M, Gordon G. Prospective evaluation of the PleurX catheter when used to treat malignant ascites associated with malignancy. J Vasc Intervent Radiol 2008; 19: 1723-31.

12. Savin M, Kirsch M, Romano W, Wang S, Arpasi P, Mazon C. Peritoneal ports for treatment of intractable ascites. J Vasc Intervent Radiol 2005; 16: 363-8.

13. Ozkan O, Akinci D, Gocmen R, Cil B, Ozmen M, Akhan O. Percutaneous placement of peritoneal port-catheter in patients with malignant ascites. Cardiovasc Intervent Radiol 2007; 30: 232-6.

14. Baumann K, Pfisterer J, Wimberger P, Burchardi N, Kurzeder C, du Bois A et al. Intraperitoneal treatment with the trifunctional bispecific antibody Catumaxomab in patients with platinum-resistant epithelial ovarian cancer: A phase lla study of the AGO Study Group. Gynecol Oncol 2011; 123: 27-32.

15. Barnett T, Rubins J. Placement of a permanent tunneled peritoneal drainage catheter for palliation of malignant ascites: A simplified percutaneous approach. J Vasc Intervent Radiol 2002; 13: 379-83.

16. Akinci D, Erol B, Ciftci T, Akhan O. Radiologically placed tunneled peritoneal catheter in palliation of malignant ascites. Eur J Radiol 2008; 80: 265-8.

17. Heiss M, Murawa P, Koralewski P, Kutarska E, Kolesnik O, Ivanchenko V, et al. The trifunctional antibody catumaxomab for the treatment of malignant ascites due to epithelial cancer: results of a prospective randomized phase II/III trial. Int J Cancer 2010; 127: 2209-21. 